\title{
The wave forms of temporomandibular joint sound clicking and crepitation
}

\author{
S.E. WID MALM, * W.J. WILLIAMS ${ }^{\dagger}$ \& B.S. A D A M S ${ }^{\star}{ }^{*}$ Department of Biologic \& Materials Sciences; \\ ${ }^{+}$Department of Electrical Engineering and Computer Science; and ${ }^{\ddagger}$ Department of Pediatrics, Section of Pediatric Rheumatology, University of \\ Michigan, Ann Arbor, MI, U.S.A.
}

SUMMARY The aim of the present study was to determine the sound wave forms which correspond to auscultatory findings of temporomandibular joint (TMJ) clicking and crepitation. Such knowledge is important when selecting parts of digital recordings for spectral analysis. Electronic digital recordings were made with a sampling rate of $44100 \mathrm{~Hz}$ from 60 subjects, including 51 patients referred for suspected rheumatological disease and nine healthy subjects. Accelerometers with the bandwidth $20-3600 \mathrm{~Hz}$ were used for all subjects and complementary recordings were made from a subgroup of nine subjects using a measurement microphone with the bandwidth 20-20000 Hz. The clicking sounds could be classified into different types according to differences in temporal period duration $(T)$ as measured on the analogue display. One type of clicking, found in $51 \%$ of the patients, had a $T$ of 2-20 ms. Another type, found in $70 \%$ of the subjects, had a $T$ of less than $1 \mathrm{~ms}$, often as low $\mathbf{0 . 2} \mathrm{ms}$. This type of clicking was not seen at all in the analogue display if the sampling rate was below $3000 \mathrm{~Hz}$. The character of the two types of clicking differed: the short duration sounds had a very high pitch, while the pitch of the longer duration sound was lower. Crepitation was found in $63 \%$ of the subjects and was observed to be composed of a series of short duration sounds, occurring with brief (less than $10 \mathrm{~ms}$ ) intervals. It is concluded that the accelerometer (or microphone) bandwidth should cover the entire audible range $(20-20000 \mathrm{~Hz})$, and that sampling rates must be much higher than $3000 \mathrm{~Hz}$, and preferably greater than $10000 \mathrm{~Hz}$, before the true significance of electronically recorded joint sounds/ vibrations can be determined.

\section{Introduction}

The recording of temporomandibular joint (TMJ) sounds is an important diagnostic aid in clinical examination of patients with suspected TMJ disorders (Watt, 1963; Farrar \& McCarty, 1983). Electronic recording of TMJ sounds and vibrations permits a more detailed analysis and provides more reliable comparisons among different observers and among different examination sessions (Ekensten, 1952; Widmalm \& Hedegård, 1974; Katzberg $\&$ Westesson 1993). Such examination usually involves a comparison of sounds heard on auscultation with spectral analysis of corresponding parts of electronic recordings (Oullette, 1974; Heffez \& Blaustein, 1986; Drum \& Litt, 1987; Gay \& Bertolami, 1987; Christensen, Donegan
\& McCay, 1992; Ishigaki, Bessette, \& Maruyama, 1993). The selection of portions of the electronic record for spectral analysis often follows visual inspection of the displayed signal, and usually includes deflection indicating the presence of sound/vibration recording. The form of those deflections can be expected to be different for different types of sounds, and to be affected by damping factors in the tissues between the source of sound and the recording device. Watt's pioneering work (1963), based on analogue sound recordings of TMJ sounds, described the wave forms of clicking and crepitus as seen on oscilloscopic tracings. Little, if anything, has been added to our knowledge about the association between different wave forms and types of TMJ pathology since that time. Nor do we know how the sound wave forms are affected 
by the choice of recording technology, such as the sampling rate. This issue is of particular importance because much of the current research in this area is based upon spectral analysis of digital sound recording selected on the basis of visual inspection of visible wave forms.

It is of crucial importance to separate artefacts from biological signals when using electrophysiological recording systems to investigate a biological process (Widmer, 1989; Mohl et al., 1990). A previous study has shown that TMJ sound recordings often include sounds with very high frequencies, such that a sampling rate of $7200 \mathrm{~Hz}$ or lower does not avoid aliasing (Widmalm et al., 1996). The aim of the present study was to determine the wave forms of clicking and crepitation recorded with a sampling rate $(44100 \mathrm{~Hz})$ which is standard for high fidelity speech and sound recording, and which should capture the wave forms of all audible TMJ sounds. By using lower sampling rates, we have also tested their effects on the quality of TMJ sound recordings.

\section{Materials and methods}

\section{Subjects}

The group consisted of 51 patients, $34(67 \%)$ females and $17(33 \%)$ males, referred for suspected rheumatologic disease to the Pediatric Rheumatology Clinic at the University of Michigan Medical Center. The mean age was $12 \cdot 3 \pm 3.99$ (s.d.) years (range 4-20). A group of nine healthy subjects (age range 6-32) were also included for control recordings with a measurement microphone. The patients were questioned about presence of temporomandibular joint (TMJ) sounds and were examined for TMJ sounds by auscultation using a stethoscope and by electronic recording. Sounds were noted as present if they were observed in any joint during at least two of 12 opening cycles.

\section{Electronic recording}

Recordings were made during 12 habitual maximal opening and closing movements starting and ending in rest position without making tooth contact. Piezoelectric accelerometers*, which measure acceleration of the rate at which the velocity of the vibrating tissues is changing, were used to record the sounds. The accelerometers exhibited a resonant frequency at $3800 \mathrm{~Hz}$ and a flat response between 20 and $3600 \mathrm{~Hz}$. The accelerometers

* Model 207A, Bioresearch, Milwaukee, WI, U.S.A. were fastened over the lateral aspects of the TM joints and held in place by a spring holder. Recordings were also made in a control group of nine subjects with a measurement electret condenser microphone ${ }^{\dagger}$ with a flat response between $20 \mathrm{~Hz}$ and $20000 \mathrm{~Hz}$.

The sounds were digitized with a sampling rate of $44100 \mathrm{~Hz}$ and recorded on tape using a two channel digital tape recorder $(\mathrm{DAT})^{\ddagger}$ and a mixer ${ }^{\S}$ with adjustable gain. The DAT had the following specifications: quantization was 16 bit linear, which means that the analogue to digital conversion $(\mathrm{ADC})$ resolution was 16-bit. The total number of possible digital combinations or counts for the $\mathrm{ADC}$ was thus $2^{16}=65536$. Frequency response at recording was $1-22000 \mathrm{~Hz} \pm 0 \cdot 5 \mathrm{~dB}$. Signal-to-noise ratio, dynamic range and channel separation were better than $94 \mathrm{~dB}$. Total harmonic distortion was less than $0.004 \%$ at $1 \mathrm{kHz}$.

The recorded signals were displayed in the time domain using a Quadra 700 computer** with an Audio Media II-MM005 board"+. They were also played back and listened to using headphones and loud speakers for comparison of their character with the character of the sounds heard at auscultation.

\section{Time-frequency $(t-f)$ representation of signals}

The digitized analogue data of the TMJ sounds were transformed from the time domain to the $t-f$ domain and displayed as three-dimensional reduced interference (RID) graphs (Widmalm, Williams \& Zheng, 1991).

The effect of sampling with low sampling rates was tested by down-sampling the recorded sounds. That is, copies of the sound files were converted by taking every second or fourth of eighth, etc., sample and storing the samples in a new file. The use of a DAT makes it possible to make multiple copies without noticeable degradation of the sound quality.

\section{Results}

Auscultation and interview

Auscultation revealed clicking in $38 \%$ of patients and crepitation in $66 \% ; 42 \%$ admitted to hearing sounds (earnoise) from their jaw joints.

\footnotetext{
${ }^{\dagger}$ MI-1231, Ono Sokk Co., Tokyo, Japan.

‡ Tascam DA-30, Tascam TEAC Professional Division, Montebello, CA, U.S.A.

$\$$ Mackie, Micro Series 1202, Mackie Designs, Woodinville, WA, U.S.A.

** Apple Computer Inc., Cupertino, CA, U.S.A.

${ }^{+t}$ Digidesign Inc., Menlo Park, CA, U.S.A.
} 


\section{Electronic recording}

It was found that clicking sounds could be divided into separate groups with distinctly different temporal period duration $(T)$ as judged from the analogue display. One group of clicking sounds had a $T$ mostly well above 2-5 ms (Fig. 1). Those sounds were observed in $51 \%$ of the subjects. The number of visible oscillations were seldom more than 1-3.

A second group of clicking sounds, found in $70 \%$ of the subjects, had a $T$ of much less than $1 \mathrm{~ms}$. The analogue representation had the character of an exponentially damped sinusoid (Fig. 2). The amplitude decreased within a few ms to noise level. The sound character differed between the two types of clicking. The short duration sounds always had a very high pitch, like the snap of a dry twig breaking, while the pitch of the second type sounded lower, as judged by listening to repeated play backs.

Crepitation was recorded in $63 \%$ of the patients. The analogue display revealed that a typical crepitation was composed of a large number of short duration (less than $1 \mathrm{~ms}$ ) clicks occurring in rapid succession (Fig. 3) with short time intervals (Fig. 4). The amplitude was mostly low and the sound character was that of soft crepitus. A few patients had what is usually referred to as course crepitus or crackling (Watt, 1963, 1980; Dworkin et al., 1990a, 1990b; Michet \& Hunder, 1993). In those cases the amplitude of each click in the series was high, as high as in loud clicking audible without the aid of a stethoscope.

A display of the time-frequency distribution of the sounds with $T$ less than 1 ms (Fig. 5), recorded with the microphone with bandwith 20-20 $000 \mathrm{~Hz}$, showed that those sounds had frequency content well above $3000 \mathrm{~Hz}$ (Fig. 6).

The effect of lowering the sampling rate was crucial for the analogue representation of the snapping sounds with $T$ about $0 \cdot 2 \mathrm{~ms}$. They were recognizable as long as the sampling rate was at least about $10000 \mathrm{~Hz}$ (Fig. 7), but they were not visible in the display. when the sampling rate was below $3 \mathrm{kHz}$ (Fig. 8). The effect of lowering the sampling rate from $44100 \mathrm{~Hz}$ to about $1300 \mathrm{~Hz}$, on the display of clicking sounds with wave forms with $T$ 5-10 ms or more, was not visible by the naked eye.

\section{Discussion}

Electronic recording has been suggested as a valuable complement to auscultation of temporomandibular joint (TMJ) sounds. Such examination mostly involves spectral analysis of short sequences, usually with a duration of 100-300 ms, selected from digital recordings of whole jaw opening-closing cycles, where deflections in the analogue display indicate the presence of sounds or vibrations. Although published reports have used sampling rates in the range of $1000-3000 \mathrm{~Hz}$, which may be high enough for clicking sounds with a temporal period duration ( $T$ ) of 5-10 ms, we have found that frequently occurring TMJ sounds can be expected to have a substantial amount of energy in a frequency range well above $3000 \mathrm{~Hz}$ (Fig. 6). In fact, a majority of our subjects had clicking sounds, audible on auscultation, with such a short temporal period duration that they were not visible in the analogue display of digital

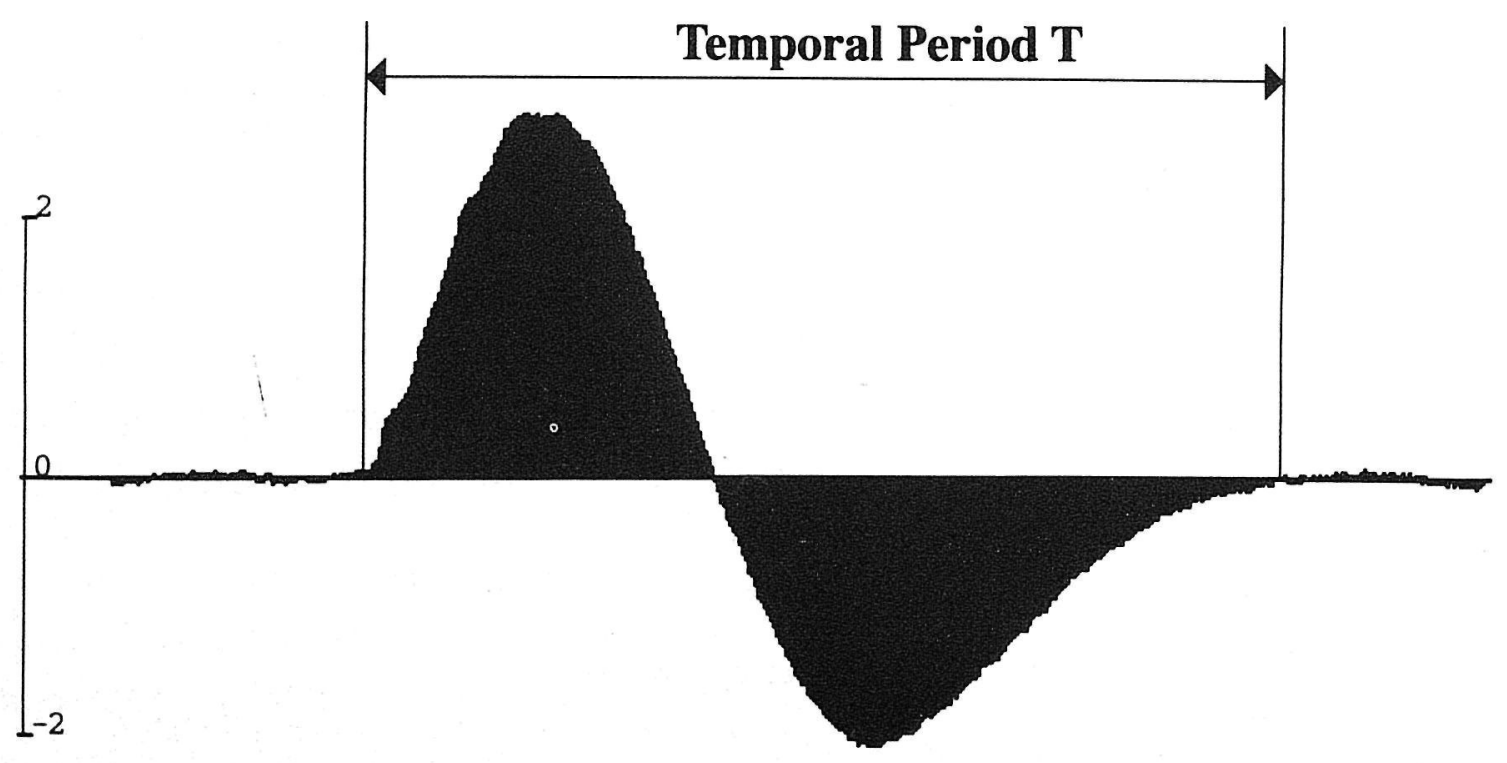

$10 \mathrm{~ms}$
Fig. 1. The wave form of a typical 'long duration' clicking. Amplitude level is in arbitrary units. 
Fig. 2. The wave form of a typical short duration clicking (snapping). Note the exponential decay of amplitude down to noise level (arrow E) after about 2 ms. $T=$ temporal period duration. Amplitude level is in arbitrary units.
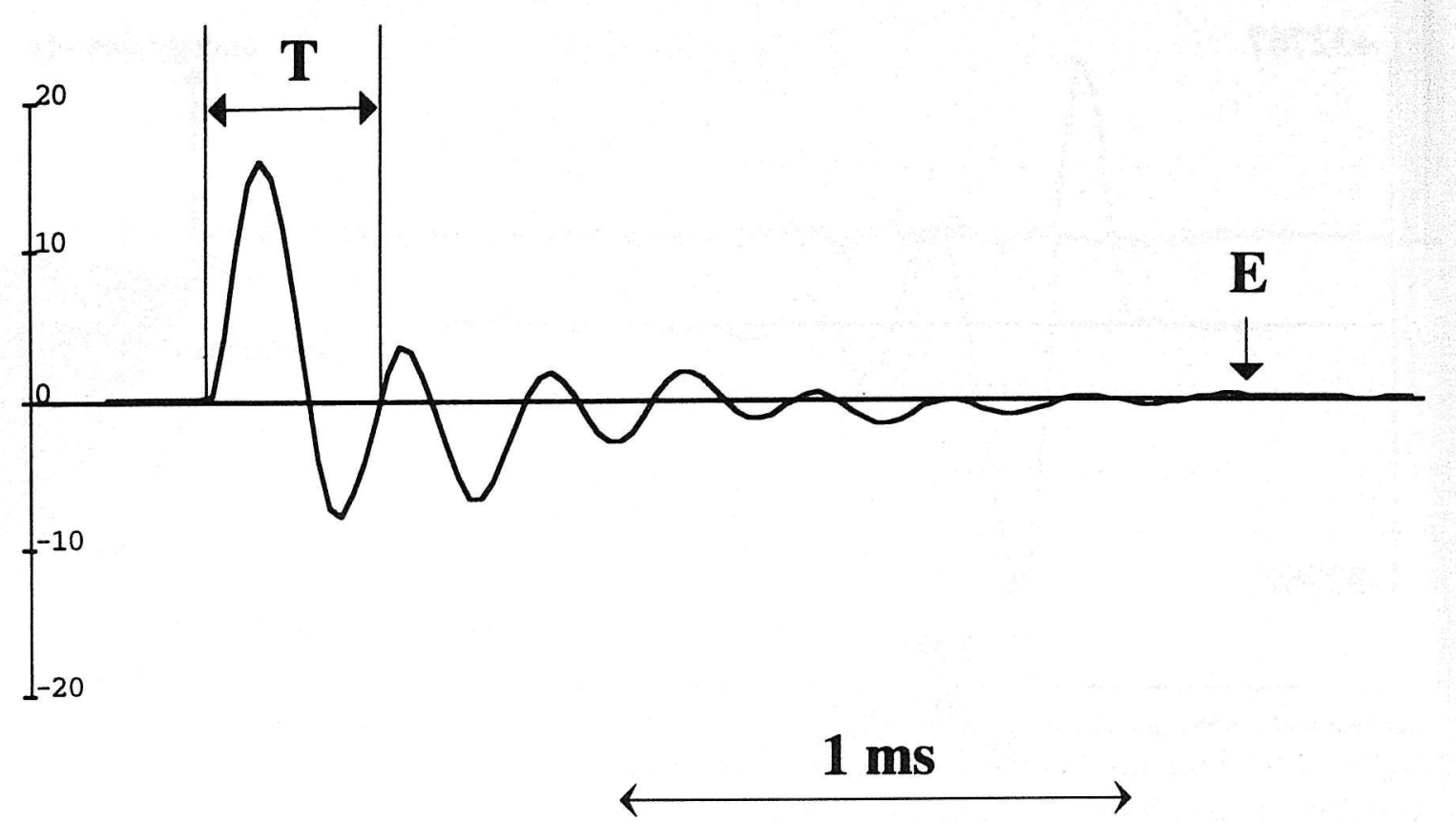

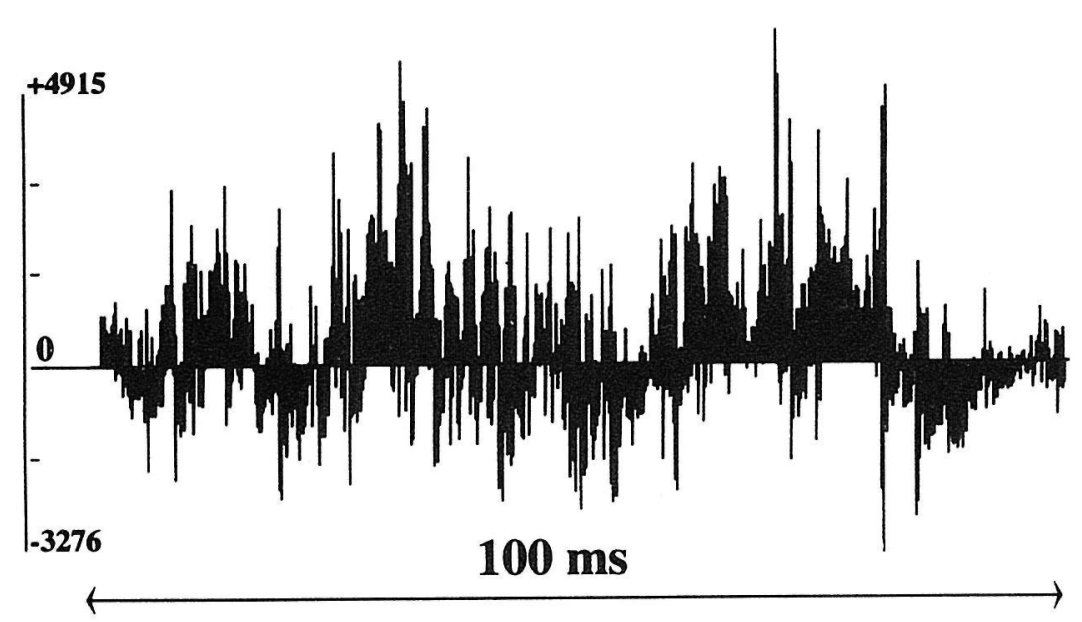

Fig. 3. Crepitation recording. Note the multitude of spikes each one representing one snapping sound like the clicking in Fig. 2. Amplitude level is in arbitrary units. The amplitudes were low and the sound character was that of soft crepitus. When a series of clicks occurred with high amplitude, the sound character was that of coarse crepitus or crackling. Vertical scale: quantized sample value (full scale +32767 to -32767 ).

recordings unless the sampling rate was approximately $10000 \mathrm{~Hz}$ or more (Figs 7 \& 8).

Ordinarily, data are passed through an 'anti-aliasing filter' which is set to pass only signal components below frequencies which are, perhaps one-third to onequarter of the sampling frequency. The well known sampling theorem requires that signal components above one-half the sampling frequency be removed. Thus, if the sampling frequency was $3000 \mathrm{~Hz}$, the antialiasing filter, if properly applied, would severely attenuate all signal components above about $1000 \mathrm{~Hz}$. As can be seen from Fig. 6, the greater part of the energy of the signal would be removed by such filtering. We believe that signal components of critical value to the diagnostic capability of these signals may

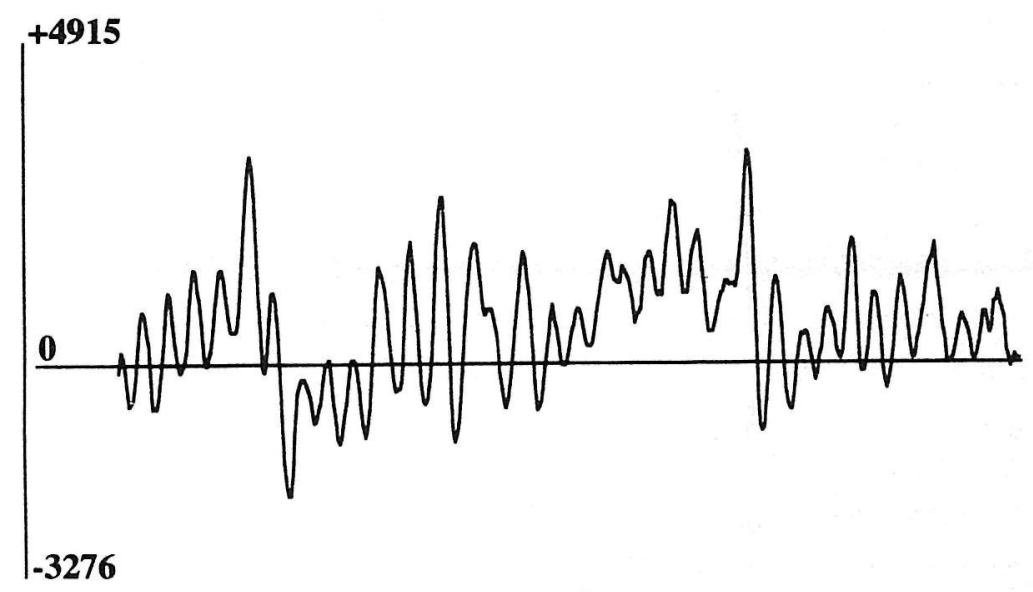

$10 \mathrm{~ms}$

Fig. 4. A portion of the crepitation recording of Fig. 3 shown with high time resolution. Note that a number of short duration clicks occur close in time. Vertical scale: quantized sample value (full scale +32767 to -32767$)$.

be removed by such filtering. This may explain why some investigators have found the frequency content above $650 \mathrm{~Hz}$ to be negligible (Ishigaki et al., 1993; Tallents et al., 1993). If a proper anti-aliasing filter was used in these studies, then it is possible that important signal components above $650 \mathrm{~Hz}$ were removed by this filter. We would conclude rather that sampling rates must be much higher than $3000 \mathrm{~Hz}$ if all joint sounds and vibrations are to be considered. When sampling rates less than $3000 \mathrm{~Hz}$ are used to compare auscultatory findings with electronic recordings, confusion may result because some audible sounds may not be visible in the electronic recording, while inaudible low frequency vibration may cause distinct deflections. 


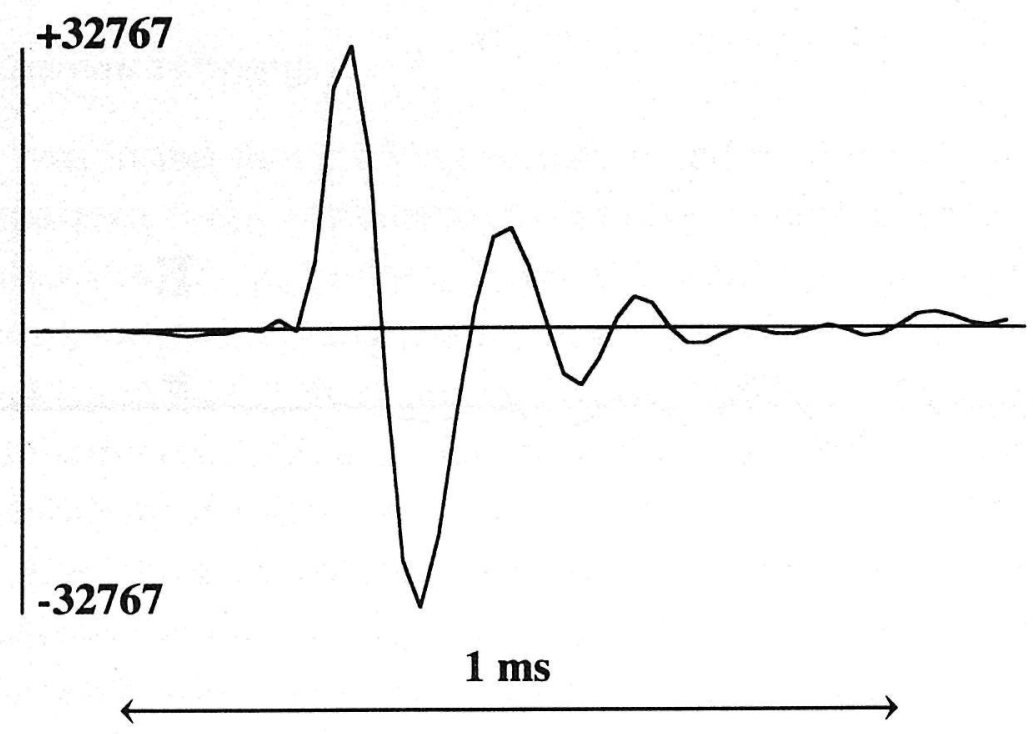

Fig. 5. Short duration click with a temporal period duration of $0 \cdot 2 \mathrm{~ms}$ recorded with a microphone with a flat response from 20 to $20000 \mathrm{~Hz}$. Vertical scale: Quantized sample value (full scale +32767 to -32767 ).

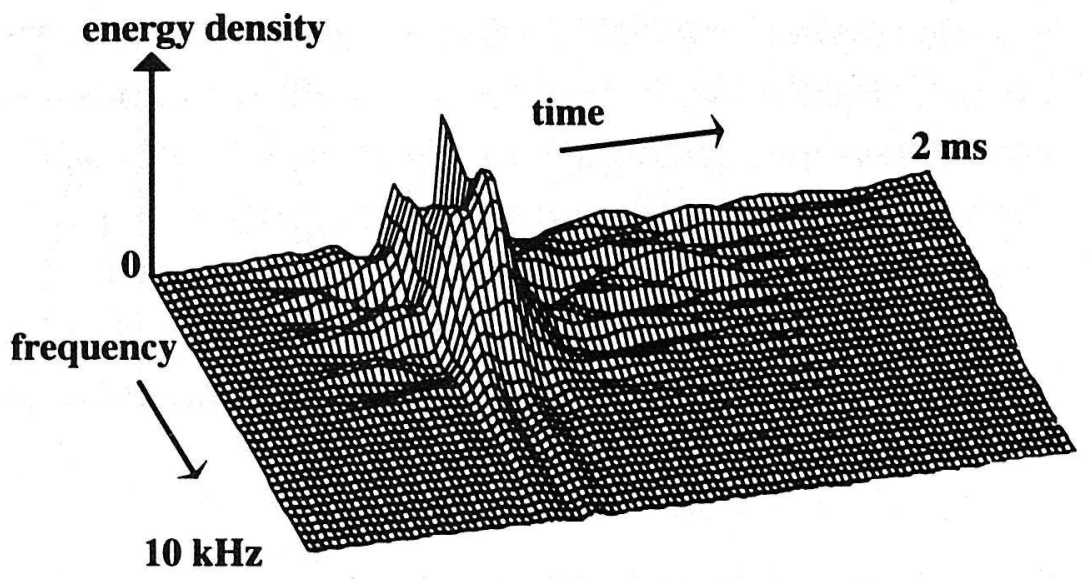

Fig. 6. Reduced interference distribution (RID) of the click in Fig. 5 displayed as a three dimensional graph (so called mesh plot). Note that a large amount of energy is located well above $3000 \mathrm{~Hz}$.
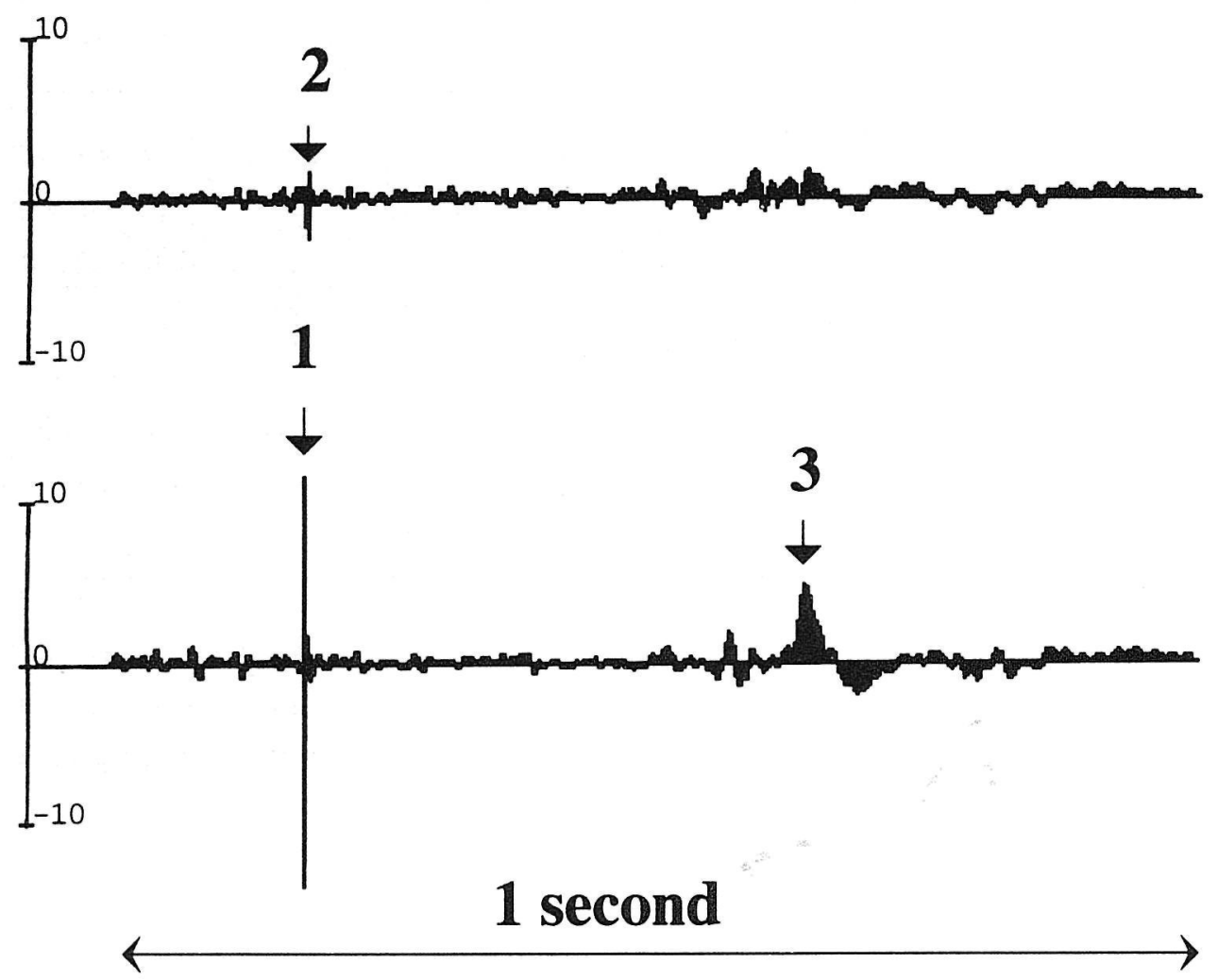

Fig. 7. Recording made with a sampling rate of $44100 \mathrm{~Hz}$. A clicking with short period duration (arrow 1) and a clicking with long period duration (arrow 3) are recorded from the left temporomandibular joint (TMJ) in the lower channel. The short duration click is also recorded in the upper channel (arrow 2). Amplitude level is in arbitrary units.

In conclusion, electronic recording can be used to separate TMJ sounds into different types of clicking and crepitation, according to temporal period duration and frequency content. Auscultation alone cannot classify these sounds in a similar manner, nor can auscultatory findings of different examiners be reliably documented for comparison. It is important to note that the majority of TMJ sounds may occur with frequencies well above $3000 \mathrm{~Hz}$, and that for analogue display of digital recordings sampling rate should be at least $10000 \mathrm{~Hz}$. The standard sampling rate for high fidelity speech and music recording, $44100 \mathrm{~Hz}$, in conjunction with high quality accelerometers or microphones with a flat response from 20 to $16000-20000 \mathrm{~Hz}$, may be most appropriate to ensure true reproduction of TMJ sounds on playback.

\section{Acknowledgments}

The authors express their thanks to Steve Shoemake and Andrew Chang for their programming work. This study was supported in part by a grant from the Office of the Vice President of Research, University of Michigan, Ann Arbor, MI and from the National Science Foundation, NSF grant no. BCS 9110571. 


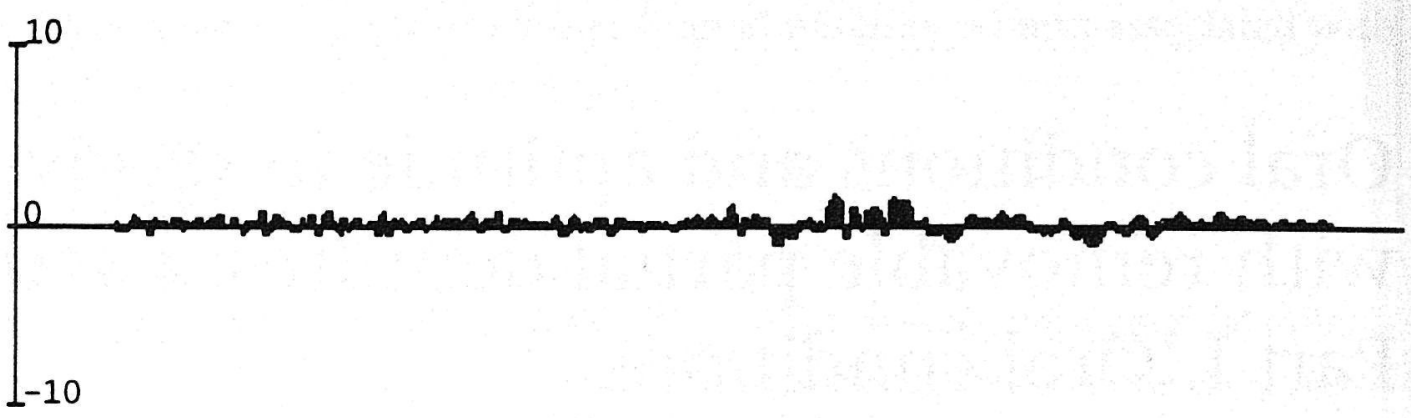

Fig. 8. Sound recording in Fig. 7 shown after resampling with $2756 \mathrm{~Hz}$ but with the same amplification. Note that the short duration clicking (arrows 1 and 2 in Fig. 7) is not visible. The duration of the spike portion of the short duration clicking was about $1 \mathrm{~ms}$ and the sampling rate had to be about $10000 \mathrm{~Hz}$ to 'catch' it. Amplitude level is in arbitrary units.

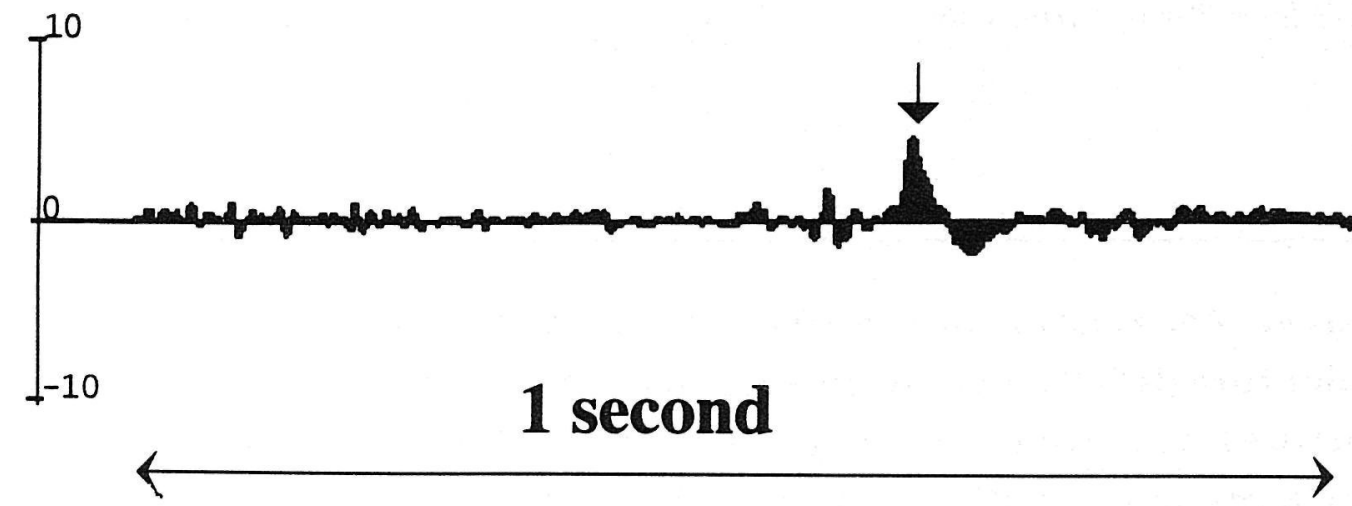

\section{References}

Christensen, L.V., Donegan, S.J. \& McCay, D.C. (1992) Temporomandibular joint vibration analysis in a sample of non-patients. Journal of Craniomandibular Practice, 10, 35.

Drum, R. \& LrTT, M. (1987) Spectral analysis of temporomandibular joint sounds. Journal of Prosthetic Dentistry, 58, 485.

Dworkin, S.F., Huggins, K.H., LeResCHe, L. et al. (1990a) Epidemiology of signs and symptoms in temporomandibular disorders: clinical signs in cases and controls. Journal of American Dental Association, 120, 273.

Dworkin, S.F., LeResche, L., DeRouen, T. \& Von KorfF, M. (1990b) Assessing clinical signs of temporomandibular disorders: Reliability of clinical examiners. Journal of Prosthetic Dentistry, 63, 574.

EKENSTEN, B. (1952) Phonograms of anomalies of the temporomandibular joint in motion. Odontologisk Tidskrift, 60, 235.

Farrar, W.B. \& McCarty JR., W.L. (1983) In: A Clinical Outline of Temporomandibular Joint Diagnosis and Treatment, 7th edn, pp. 53-88. Normandie Publications, Montgomery, Alabama.

GAY, T. \& BeRTolami, C.N. (1987) The spectral properties of temporomandibular joint sounds. Journal of Dental Research, 66, 1189.

Heffez, L. \& Blaustein, D. (1986) Advances in sonography of the temporomandibular joint. Oral Surgery, Oral Medicine, Oral Pathology, 62, 486.

Ishigaki, S., Bessette, R.W. \& MAruyama, T. (1993) A clinical study of temporomandibular joint (TMJ) vibrations in TMJ dysfunction patients. The Journal of Craniomandibular Practice, 11, 7.

Katzberg, R.W. \& Westesson, P.L. (1993) In: Diagnosis of the Temporomandibular Joint. pp. 241-249. W.B. Saunders Co, Philadelphia.

Michet, C.J. \& Hunder, G.G. (1993) Examination of the joints. In: Textbook of Rheumatology. Eds. W.N. Kelley, E.D. HARris JR., S. Ruddy \& C.B. Sledge. p. 353 . W.B. Saunders, Philadelphia.
MoHL, N.D., Lund, J.P., WidMeR, C.G. \& McCALI, W.D. JR. (1990) Devices for the diagnosis and treatment of temporomandibular disorders. Part II: Electromyography and sonography. The Journal of Prosthetic Dentistry, 63, 332.

OuelLetTE, P.L. (1974) TMJ sound prints: electronic auscultation and sonographic audiospectral analysis of the temporomandibular joint. Journal of American Dental Association, 89, 623.

Tallents, R.H., Hatala, M., Katzberg, R.W. \& Westesson, P.L. (1993) Temporomandibular joint sounds in asymptomatic volunteers. Journal of Prosthetic Dentistry, 69, 298.

WATT, D.M. (1963) A preliminary report on the auscultation of the masticatory mechanism. The Dental Practitioner, 14, 27.

WATT, D.M. (1980) Temporomandibular joint sounds. Journal of Dentistry, 8, 119.

WidMALM, S.-E. \& HEDEGÅRD, B. (1974) An apparatus for the synchronous registration of EMG activity in jaw muscles and of vibrations in the masticatory system. Journal of Oral Rehabilitation, 1, 183.

Widmalm, S.E., Williams, W.J., \& Zheng, C. (1991) Time frequency distributions of TMJ sounds. Journal of Oral Rehabilitation, 18, 403.

Widmalm, S.E., Williams, W.J., Christiansen, R.L., GunN, S.M. \& PARK, D.K. (1996) Classification of temporomandibular joint sounds based upon their reduced interference distribution. Journal of Oral Rehabilitation, 23, 35.

Wmmer, C.G. (1989) Temporomandibular joint sounds: a critique of techniques for recording and analysis. The Journal of Craniomandibular Practice, 7, 213.

Correspondence: Dr Sven E. Widmalm, 1565 Kuehnle, Ann Arbor, MI 48103, U.S.A. 
This document is a scanned copy of a printed document. No warranty is given about the accuracy of the copy. Users should refer to the original published version of the material. 